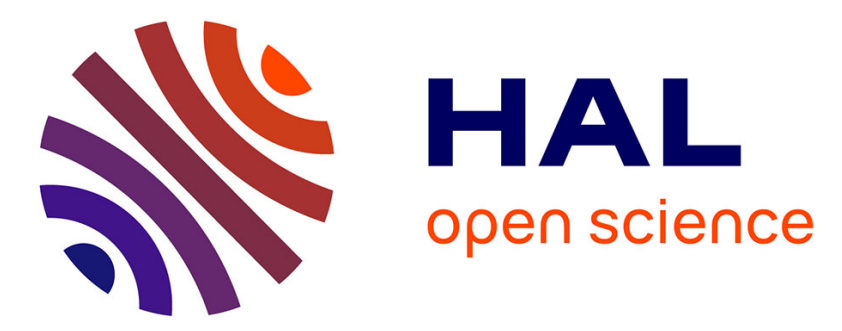

\title{
Comparative study on the performance of multiparameter SAR Data for operational urban areas extraction using textural features
}

C. Corbane, N. Baghdadi, Xavier Descombes, G.J. Wilson, N. Villeneuve, Michel Petit

\section{To cite this version:}

C. Corbane, N. Baghdadi, Xavier Descombes, G.J. Wilson, N. Villeneuve, et al.. Comparative study on the performance of multiparameter SAR Data for operational urban areas extraction using textural features. IEEE Geoscience and Remote Sensing Letters, 2009, 6 (4), p. 728 - p. 732. 10.1109/LGRS.2009.2024225 . hal-00456174

\section{HAL Id: hal-00456174 https://hal.science/hal-00456174}

Submitted on 12 Feb 2010

HAL is a multi-disciplinary open access archive for the deposit and dissemination of scientific research documents, whether they are published or not. The documents may come from teaching and research institutions in France or abroad, or from public or private research centers.
L'archive ouverte pluridisciplinaire HAL, est destinée au dépôt et à la diffusion de documents scientifiques de niveau recherche, publiés ou non, émanant des établissements d'enseignement et de recherche français ou étrangers, des laboratoires publics ou privés. 


\title{
Comparative Study on the Performance of Multiparameter SAR Data for Operational Urban Areas Extraction Using Textural Features
}

\author{
Christina Corbane, Nicolas Baghdadi, Xavier Descombes, Geraldo Wilson Junior, \\ Nicolas Villeneuve, and Michel Petit
}

\begin{abstract}
The advent of a new generation of synthetic aperture radar (SAR) satellites, such as Advanced SAR/Environmental Satellite (C-band), Phased Array Type L-band Synthetic Aperture Radar/Advanced Land Observing Satellite (L-band), and TerraSAR-X (X-band), offers advanced potentials for the detection of urban tissue. In this letter, we analyze and compare the performance of multiple types of SAR images in terms of band frequency, polarization, incidence angle, and spatial resolution for the purpose of operational urban areas delineation. As a reference for comparison, we use a proven method for extracting textural features based on a Gaussian Markov Random Field (GMRF) model. The results of urban areas delineation are quantitatively analyzed allowing performing intrasensor and intersensors comparisons. Sensitivity of the GMRF model with respect to texture window size and to spatial resolutions of SAR images is also investigated. Intrasensor comparison shows that polarization and incidence angle play a significant role in the potential of the GMRF model for the extraction of urban areas from SAR images. Intersensors comparison evidences the better performances of $\mathrm{X}$-band images, acquired at 1-m spatial resolution, when resampled to resolutions of 5 and $10 \mathrm{~m}$.
\end{abstract}

Index Terms-Gaussian Markov random field (GMRF) model, synthetic aperture radar (SAR), urban remote sensing.

Manuscript received February 26, 2009; revised April 28, 2009. This work was supported by the European Union. It was conducted within the framework of GMES/LIMES project (Land and Sea Integrated Monitoring for European Security).

C. Corbane is with the Institut de Recherche pour le Développement, ESPACE Unit, Maison de la télédétection, 34093 Montpellier, France, and also with the Joint Research Centre (JRC), Institute for the Protection and Security of the Citizen (IPSC), 21027 Ispra (VA), Italy (e-mail: corbane@ teledetection.fr).

N. Baghdadi is with the Centre National du Machinisme Agricole, du Génie Rural, des Eaux et Forêts (CEMAGREF), UMR TETIS-Territoires, environnement, télédétection et information spatiale, 34093 Montpellier, France.

$\mathrm{X}$. Descombes is with the Institut National de Recherche en Informatique et en Automatique (INRIA), 06902 Sophia Antipolis Méditerranée, France.

G. W. Junior is with the Instituto Alberto Luiz Coimbra de Pos-Graduação e Pesquisa de Engenharia (COPPE-UFRJ), Graduate School and Research in Engineering, Federal University of Rio de Janeiro, and CAPES, Center for the Enhancement of Graduate Level Personnel, Rio de Janeiro, RJ 21945-970, Brazil.

N. Villeneuve is with the Institut de Recherche pour le Développement, ESPACE Unit, Moufia University Campus, Sainte-Clotilde, 97492 Reunion Island, France.

M. Petit is with the Institut de Recherche pour le Développement, ESPACE Unit, Maison de la télédétection, 34093 Montpellier, France.

Color versions of one or more of the figures in this paper are available online at http://ieeexplore.ieee.org.

Digital Object Identifier 10.1109/LGRS.2009.2024225

\section{INTRODUCTION}

A ATELLITE remote sensing offers an effective solution $\checkmark$ to mapping settlements and monitoring urbanization at a range of spatial scales. Synthetic aperture radar (SAR) technology, being indifferent to weather and illumination conditions, ensures permanent monitoring. With the development of new (SAR) sensors, with high spatial resolution, multiple polarizations capabilities, and short revisit time, monitoring of urban areas by means of SAR images has grown into a valuable and indispensable tool. Depending on the task, the requirements concerning resolution, precision, coverage, reliability, actuality, etc., are very different and are crucial for the optimal sensor configuration.

SAR research with a clear focus on urbanized areas was begun in the late 1960s [1], [2]. Bryan [3] and Henderson [4] have carried out most extensive research on issues related to urban land use mapping from SAR data. A comprehensive review of SAR applications in urban studies is given in [5]. The individual benefits of medium spatial and high spatial resolution SAR imagery and SAR-derived texture measures for settlement mapping have been widely extolled (e.g., [6] and [7]). However, very few studies have attempted to compare the performances of multiparameter systems [8]. Bryan [3] and Haack [9] assessed the influence of wavelength in urban features identification. The effect of polarization on the interpretation of urban features was studied in [8], [10], and [11]. As for SAR incidence angle, it is known that this acquisition parameter affects feature identification in an urban environment through its control of range resolution, image layover, and radar shadows. Comparisons of the effects of incidence angles on urban areas detection were reported in [12] and [13]. To our knowledge, no studies have fully analyzed and compared different types of SAR images in terms of frequency, polarization, incidence angle, and spatial resolution for urban mapping.

The motivation underlying this letter is to investigate performance of multiparameter SAR sensors for operational urban areas delineation using a proven automatic approach based on a Gaussian Markov random field (GMRF) model. This approach has already demonstrated its suitability for operational rapid urban mapping in [14]. It is particularly designed to exploit the spatial characteristics of medium to high spatial resolution images by transforming gray level information into texture information. This is convenient when looking at high-density urban areas as well as at medium to large-scale features, like the 
TABLE I

Multiparameter SAR Data Set Available for the COMMUNE OF SAINTE-MARIE (REUNION ISLAND)

\begin{tabular}{l|cc|ccc|cc}
\hline \hline & \multicolumn{2}{|c|}{ ASAR } & \multicolumn{3}{c|}{$\begin{array}{c}\text { PALSAR } \\
\text { (C-BAND) }\end{array}$} & \multicolumn{2}{c}{$\begin{array}{c}\text { TerraSAR-X } \\
\text { (X-band) }\end{array}$} \\
\hline Date & Nov & Jan & Sept & Feb & Aug & Jan & Jan \\
& 11 & 16 & 21 & 20 & 06 & 14 & 13 \\
& 2007 & 2008 & 2007 & 2007 & 2007 & 2008 & 2008 \\
Polarization & $\mathrm{HH}$ & $\mathrm{HH}$ & $\mathrm{HH}$ & $\mathrm{HH}$ & $\mathrm{HH}$ & $\mathrm{HH}$ & $\mathrm{HH}$ \\
Incidence & $\mathrm{VV}$ & $\mathrm{VV}$ & & $\mathrm{HV}$ & $\mathrm{HV}$ & $\mathrm{H}$ & \\
angle $\left(^{\circ}\right)$ & 14 & 30 & 38 & 44 & 38 & 52 & 32 \\
& & & & & & & \\
Pixel size $(\mathrm{m})$ & 12.5 & 12.5 & 6.25 & 12.5 & 12.5 & 1 & 1 \\
\hline
\end{tabular}

boundaries between urban and nonurban areas. The method is tested on a set of C- and L-band and single-channel X-band images acquired at different polarizations over the town of Sainte Marie in the Reunion Island. To investigate the optimal sensors configuration for automatic urban areas delineation using the GMRF model, different subsets are considered, composed of single-polarization-channel SAR data with different spatial resolutions and incidence angles. The data set allows performing intrasensor and intersensors comparisons and analyzing the sensitivity of the GMRF model with respect to texture window size and to spatial resolutions of SAR images. The results are quantitavely analyzed by considering classification accuracy and the percentage of false alarms as measures of performance.

\section{Multiparameter SAR Data COMPARISON METHODOLOGY}

\section{A. Data Set and Study Area}

For our comparative analyses, we consider a data set of seven SAR images (Table I).

All the images are georeferenced to a SPOT-5 HRG-PAN scene used as a reference for geographic information. The presence of multiplicative speckle noise in SAR images reduces the ability of the image to distinguish urban features. A Lee sigma filter is chosen to reduce speckle effects with a window size of $5 \times 5$. To demonstrate the effect of spatial resolution on the choice of texture window size used in the GMRF model, TerraSAR-X 1-m resolution images are resampled to 5 and $10 \mathrm{~m}$ using the nearest neighbor approach. This resampling approach can be applied with a limited effect on speckle and without severe side effects such as information loss.

The image data set is available for the commune of SainteMarie on the north side of the Reunion Island, a mountainous area with cultivated land and cities concentrated on the coastal lowlands. Sainte-Marie has a population of 26582 , and a terrain elevation ranging between 0 and $1800 \mathrm{~m}$. This commune is one of the main locations presenting urban expansion in the Reunion Island in recent years, with large tracts of agricultural land being converted into built land uses.

\section{B. Overview of the GMRF Model}

For an automatic delineation of urban areas, we use a proven strategy that consists in estimating a texture parameter from a SAR image through an eight directional GMRF models. This approach was initially developed by [15], generalized in [16], and recently evaluated in [14]. We briefly recall the definition of the texture parameter. Full details can be found in [15]. This parameter is obtained from a multidirectional analysis of the texture. We consider eight directions in the discrete space and a GMRF for each direction. A pixel has two neighbors corresponding to the direction $d$ for each model.

It is shown in [16] that the conditional probability is a normal law, depending only on the mean of the 1-D neighborhood $m_{S}^{d}$

$$
P\left(X_{S} \mid m_{S}^{d}\right) \equiv N\left(\frac{2 m_{S}^{d}+\mu \lambda^{d}}{2+\lambda^{d}}, \frac{1}{2 \beta^{d}\left(2+\lambda^{d}\right)}\right)
$$

where

$d \quad$ represents the direction;

$X_{s} \quad$ is the gray level value of pixel S;

$m_{S}^{d} \quad$ average of the two neighbors of $\mathrm{S}$ in direction $d$ and $X_{r}$;

$\mu \quad$ local mean;

$\beta$ and $\lambda$ texture parameters of the model.

The texture descriptor we consider is given by the conditional variance

$$
\sigma_{P_{\left(X_{S} \mid X_{r}\right)^{d}}}^{2}=\frac{1}{2 \beta^{d}\left(2+\lambda^{d}\right)} .
$$

It is estimated by the so-called "comet tail" method in a window centered in pixel S [6]. The eight estimated values are then normalized with respect to the eight different directions in order to correct the bias introduced by the lattice anisotropy. Normalization is done by decimation that consists in computing marginal laws on sublattices [16]. The eight normalized parameters are then combined into a single one (the texture parameter) characterizing the urban areas: for each pixel, we classify the eight values in increasing order, and we only keep the mean of the two median values (even number of parameters). Indeed, these values are high for pixels inside urban areas, which are characterized by high variances in all directions. They are low for pixels in forests, fields, and water areas, which are characterized by low variances in all directions. To keep more than two median values (four or six) does not improve the detection of urban areas. The estimated texture parameter is robust and highly characteristic of urban areas. The resulting texture parameter image is finally segmented using a $K$-means algorithm allowing an accurate delineation of urban areas.

\section{Performance Evaluation of Multiparameter SAR Data}

The urban mask extraction is applied on the set of SAR images. This texture model is adapted to single-channel date, therefore the two channels of dual-polarized Advanced SAR (ASAR) and Phased Array Type L-band Synthetic Aperture Radar (PALSAR) images are processed separately. There is a tradeoff in choosing the window size for the texture analysis. Indeed, as the window size increases, the texture feature is better estimated in terms of statistics robustness, but the uncertainty area between two different textures also gets larger, and edges are not accurately localized. A window size of $15 \times$ 15 pixels has proved to be a good compromise for images with 


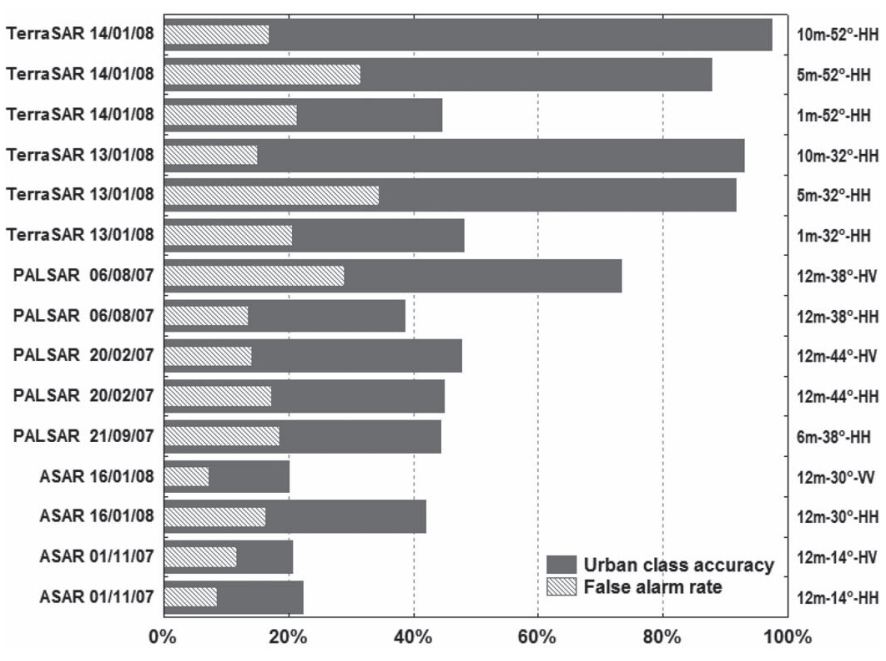

Fig. 1. Performance evaluation of multiparametric SAR data for urban areas extraction analyzed in terms of urban accuracy and false alarm.

spatial resolutions ranging between 5 and $20 \mathrm{~m}$ [17]. In order to provide a fair comparison of the results achievable with the different images, particular attention has been devoted to the choice of the reference validation set. The latter was retrieved by a photo interpretation through a manual delineation of urban areas on each image.

A small test area of $2 \mathrm{~km} \times 2 \mathrm{~km}$ is used for the evaluation of the results in terms of intrasensor (e.g., PALSAR HH against PALSAR HV polarization) and intersensors (e.g., PALSAR data against ASAR data) comparisons.

To provide a quantitative measure we consider two different accuracies: The general performance measure is provided by the urban class accuracy that represents the fraction of the pixels of the validation set that are correctly assigned to the urban cluster. The false alarm records the fraction of the pixels that are falsely classified in the urban cluster with reference to the number of urban ground-truth pixels.

\section{Statistical Performance Assessment}

\section{A. Intrasensor and Intersensors Comparisons}

Fig. 1 shows the accuracy assessment based on the error matrices produced per image. Three bars are represented for each of the two available TerraSAR-X images. They actually correspond to the 1-m images resampled to spatial resolutions of 5 and $10 \mathrm{~m}$ and that are analyzed in terms of suitability of spatial resolution for the delineation of urban areas. The two summary graphics of Fig. 1 enable intersensors as well as intrasensor comparison. As it is apparent from Fig. 1, large differences in overall accuracies are observed between the different sensors and between the images of the same sensor. We also notice that the higher the urban class accuracy, the more increasing is the false-alarm rate, as shown in Fig. 1.

In terms of intrasensors comparisons, we note that the performance of each sensor depends much more on the polarization than on the incidence angle or on the spatial resolution. This can be mainly observed for ASAR and PALSAR images acquired on January 16, 2008 and August 6, 2007, respectively.
An accuracy of $42 \%$ (with a false-alarm rate of $16 \%$ ) is obtained for HH polarization ASAR image against $20 \%$ only for VV polarization (with a false-alarm rate of 7\%). Likewise, the HV polarization of PALSAR image results in accuracy of $73 \%$ against $38 \%$ for the $\mathrm{HH}$ polarization, with a false-alarm rate of $29 \%$ against $13 \%$, respectively.

Differences in incidence angles also influence the urban areas retrieval model. This is evidenced by the better results obtained for HH polarization ASAR image (January 16, 2008) acquired with an incidence angle of $30^{\circ}$ compared to the ASAR image acquired on November 1, 2007 with the same characteristics in terms of polarization and spatial resolution, but having an incidence angle of $14^{\circ}$. As for the false-alarm rate, it is of $16 \%$ for the ASAR image at incidence angle of $30^{\circ}$ while, for an incidence angle of $14^{\circ}$, it is of $9 \%$ only.

With respect to spatial resolution, there are no significant differences between 6- and 12-m resolution PALSAR images. In contrast, a great difference in overall extraction performances can be observed between 1-m TerraSAR-X images and the same images resampled to spatial resolutions 5 and $10 \mathrm{~m}$. This implies that in X-band frequency, the spatial resolution is a critical parameter for the extraction of urban areas using the GMRF model.

In terms of intersensors evaluation, it can be noticed that the HV polarization of the L-band frequency PALSAR image acquired on August 6, 2007 yields the best accuracy result of $73 \%$ and a false-alarm rate of $29 \%$. However, when resampled to spatial resolutions of 5 and $10 \mathrm{~m}$, TerraSAR-X images outperform PALSAR whatever the incidence angle. Going from 1 to $10 \mathrm{~m}$, the classification accuracy gradually improves. However, the improvement is greater at finer resolutions. For instance, on TerraSAR-X image acquired on January 13, 2008, the improvement is more than $43 \%$ for a spatial resolution of $5 \mathrm{~m}$ with reference to $1-\mathrm{m}$ resolution and less than $1.2 \%$ for the 10-m resolution compared to 5-m resolution. So far, we noticed that the false-alarm rate increases with the urban class accuracy. However, for the 10-m resolution TerraSAR-X images, not only we get the best accuracy but also have a relatively low falsealarm rate of $15 \%$ and $17 \%$ only. From the analysis of Fig. 1, it is possible to draw some conclusions.

1) The polarization and the incidence angle play a significant role in the potential of the GMRF model for extracting urban areas from SAR images. When taking into account C- and L-bands only, the HV polarization yields the best results, and moderate incidence angles seem to be more suitable for an acceptable discrimination of urban areas.

2) X-band data provide the best overall accuracies independently of the polarization and of the incidence angle. In particular, with $10-\mathrm{m}$ resolution images, it is possible to achieve a good discrimination of urban areas with rather tolerable false-alarm rates.

3) Finally, the delineation of urban areas using the GMRF model is influenced by the spatial resolution of TerraSAR-X data. At a metric resolution, urban areas are characterized by a macrotexture more than by a microtexture. 


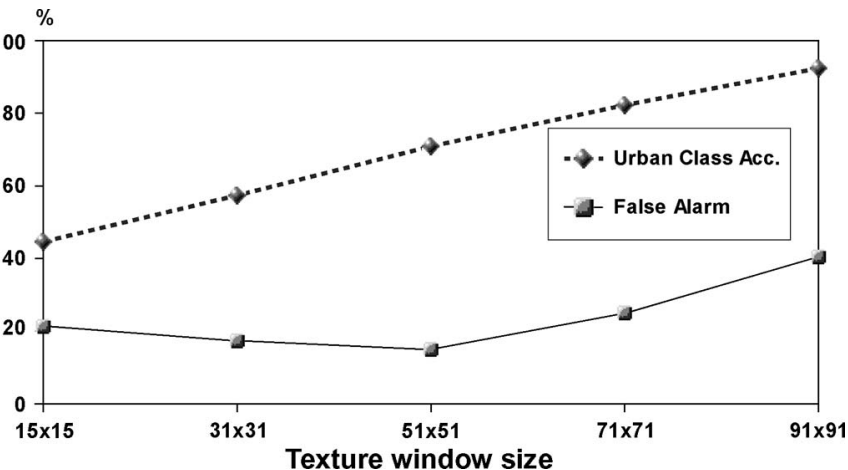

Fig. 2. Urban class accuracy and corresponding false-alarm percentages obtained for different texture window sizes with TerraSAR-X 1-m resolution data.

The obtained results suggest evaluating the effect of window size on the urban areas extraction from TerraSAR-X 1-m resolution data.

\section{B. Effect of Texture Window Size}

We now consider different window sizes for analyzing the 1-m resolution TerraSAR-X image acquired on January 13, 2008. The overall accuracies and false-alarm values are evaluated for each window size.

From Fig. 2, it is clear that increasing the window size for the 1-m resolution substantially improves the urban class accuracy. At the same time, false-alarm percentages also decrease progressively. However, for window sizes greater than $51 \times$ 51 pixels, the false-alarm percentages start to rapidly increase. Although the window sizes tested in this letter do not exceed $91 \times 91$ pixels, the use of even larger window sizes is not likely to result in significantly improved classification accuracy, considering the boundary effect. Hence, the optimum window size for the texture parameter estimation on $1-\mathrm{m}$ resolution TerraSAR-X images is $51 \times 51$ pixels. This generates an accuracy of $71 \%$ and a false alarm of $15 \%$. Even with this optimal window size, the performances of the $5 \mathrm{~m}$ and mainly of the 10 -m image processed with a window size of $15 \times 15$ pixels are still better than the original $1-\mathrm{m}$ resolution image. Fig. 3 shows examples of results of urban areas extraction on 1-, 5-, and 10-m resolution TerraSAR-images. The three images were processed with window sizes of $51 \times 51$ pixels for the $1-\mathrm{m}$ image and $15 \times 15$ for the 5- and 10-m images.

The $51 \times 51$ pixels window size is also tested on 5- and $10-\mathrm{m}$ resolution images for the estimation of the texture parameter and, consequently, the extraction of urban areas. This test is intended to evaluate the effect of window size on the performance of the urban areas retrieval model at different spatial resolutions.

Fig. 4 shows classification accuracies and false-alarm statistics for window sizes of $15 \times 15$ and $51 \times 51$ pixels at spatial resolutions of 1,5 , and $10 \mathrm{~m}$. It shows that increasing the window size improves the classification accuracy on the 1-m resolution image only while it has an opposite effect on images at 5- and 10-m: accuracy improves in about $23 \%$ for the $1-\mathrm{m}$ resolution image, while it declines in about $6 \%$ for images at 5 and $10 \mathrm{~m}$. As for the percentage of false alarms, it remains almost
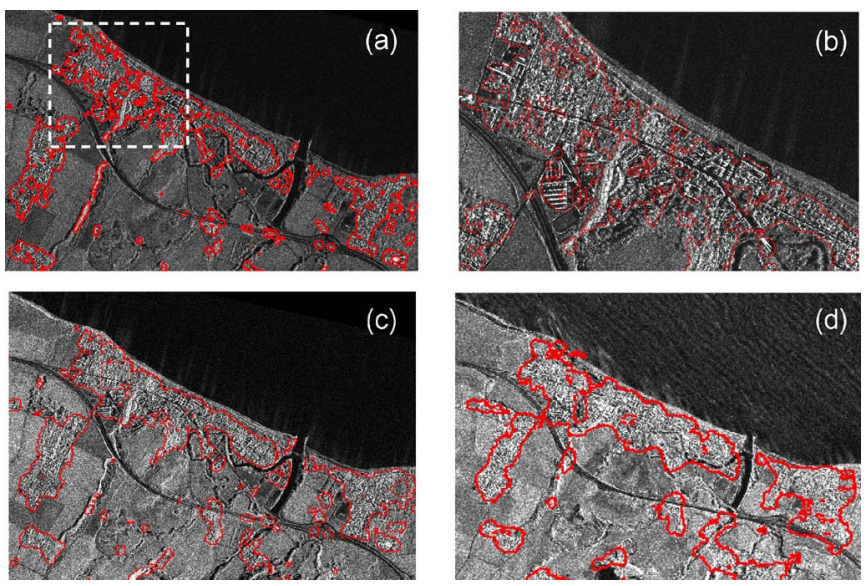

Fig. 3. Overlay of the boundaries of automatically extracted urban areas on TerraSAR-X images. (a) One-meter resolution image processed with a texture window size of $51 \times 51$ pixels. (b) Closer view of the 1 -m resolution image corresponding to the dotted square area. (c) Five-meter resolution image processed with a texture window size of $15 \times 15$ pixels. (d) Ten-meter resolution image processed with a texture window size of $15 \times 15$ pixels.

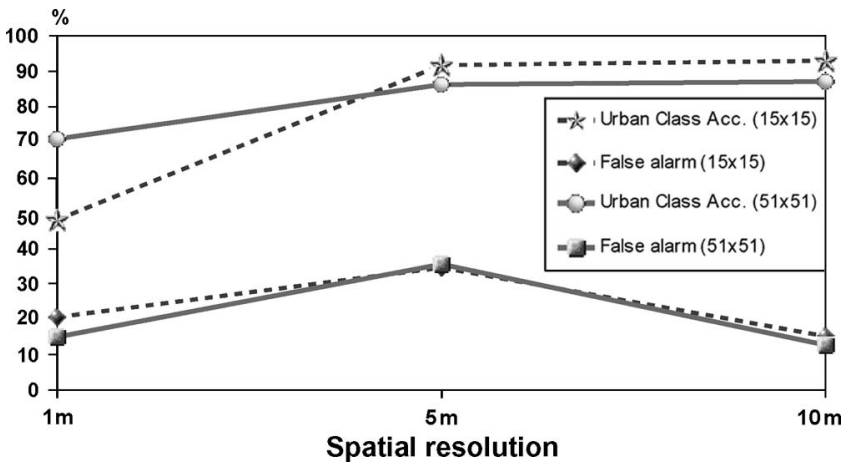

Fig. 4. Urban class accuracy and the corresponding false-alarm percentages calculated for texture window sizes of $15 \times 15$ and $51 \times 51$ pixels at spatial resolutions of 1,5 , and $10 \mathrm{~m}$ using TerraSAR-X data.

constant for 5- and 10-m resolution images and decreases by approximately $6 \%$ for the $1-\mathrm{m}$ resolution image. Thus, we conclude that for SAR images at very high spatial resolution, large texture window sizes should be used for a better identification of urban areas. The choice of the window size results of a compromise between accuracy and false alarm. As for highresolution SAR images (i.e., 5 and $10 \mathrm{~m}$ ), the choice of a window size of $15 \times 15$ is suitable for the delineation of urban areas using the GMRF model, since it provides high classification accuracies with reasonable false-alarm percentages. This confirms the results obtained by Lorette [17] who, by means of successive trials, found that a $15 \times 15$ window size was suitable for delineating urban areas on images with medium to high spatial resolutions (i.e., between 5 and $20 \mathrm{~m}$ ).

\section{CONCLUSION}

In this letter, the operational performance of multiparameter SAR images for urban areas extraction was assessed. The analysis was conducted on SAR data gathered in different frequencies (C-band, L-band, and X-band) with different spatial resolutions and incidence angles. The GMRF model which is a texture-based model designed for urban areas extraction 
served as a basis for comparison. The comparative performance analysis showed that in C-band and L-band, the results are mainly dependent on the polarization and in a lesser degree on incidence angle. The HV polarization of the L-band frequency PALSAR (L-band) image results in a high accuracy proving its relevance for urban areas delineation. This is consistent with the results of $\mathrm{Wu}$ and Sader [18] who showed that cross polarization HV is better for delineating urban features that are difficult to separate by like polarized data. SAR incidence angle also affects feature identification in an urban environment through its control of image layover and radar shadows. Our analysis indicates that moderate incidence angles produce better results than low incidence angles. Hussin [19] reached the same conclusion by demonstrating that, for accurate settlement detection and urban land cover mapping, a moderate incidence angle (around $40^{\circ}$ ) is required.

Even though the cross polarization of PALSAR image with an incidence angle of $38^{\circ}$ yields satisfactory results, our comparative analysis shows that the highest overall accuracies are obtained with TerraSAR-X images at spatial resolutions of 5 and $10 \mathrm{~m}$. These findings are consistent with the results of Bryan [3] and Haack [9] who found that X-band imagery are slightly better than L-band imagery for overall interpretation of urban feature.

Conversely, for very high spatial resolution TerraSAR-X images, we obtain relatively lower performances. These findings led us to examine the effect of texture window size on the performance of the urban areas retrieval model at different spatial resolutions. By comparing urban class accuracy and falsealarm values obtained with different window sizes, we showed that larger texture window sizes are effective in improving the classification accuracy. This finding is valid for finer spatial resolutions (1-m resolution) and in a less extent for coarser resolutions (5- and 10-m resolutions).

All of the above suggest that there is no single optimum configuration for urban areas extraction from SAR images with the GMRF model. Nevertheless, the application demonstrates that TerraSAR-X data can provide good results at spatial resolutions of 5 and $10 \mathrm{~m}$. The use of $1-\mathrm{m}$ resolution data in a texture-based model would require a large texture window size, but can also provide satisfactory results. In its turn, the GMRF model used for urban areas delineation proves to be capable of performing well for a range of spatial resolutions.

Further investigations should be conducted using a larger data set with wider ranges of incidence angles and spatial resolutions and with all possible polarizations per type of available SAR sensor. The StripMap (3-m resolution) and ScanSAR (16-m resolution) operational modes of TerraSAR-X could be used to avoid radiometric distortions and information loss induced by the downsampling approach that we applied to the original 1-m resolution images. Finally, it would be also interesting to duplicate this letter in different urban environments with various structures. This kind of analysis would particularly help in determining the optimum SAR configuration and texture window size as function of the geometrical properties of urban settings.

\section{ACKNOWLEDGMENT}

The authors would like to thank the Centre National d'Etudes Spatiales for funding the KALIDEOS database (http://kalideos.cnes.fr). The authors would also like to thank the German Space Agency, the Japan Aerospace Exploration Agency, and the European Space Agency for kindly providing TerraSAR-X (proposal HYD0007), PALSAR (AOALO.3611), and ASAR images (ASAR.351).

\section{REFERENCES}

[1] A. J. Lewis, "Evaluation of multiple polarized radar imagery for the detection of selected cultural features," NASA, Washington, DC, NASA-CR-101461, 1968.

[2] E. G. Moore, "Side looking radar in urban research," NASA, Washington, DC, Tech. Rep. NASA-138, 1969.

[3] M. L. Bryan, "Interpretation of an urban scene using multi-channel radar imagery," Remote Sens. Environ., vol. 7, pp. 49-66, 1975.

[4] F. M. Henderson, "An evaluation of Seasat SAR imagery for urban analysis," Remote Sens. Environ., vol. 12, no. 6, pp. 439-461, Dec. 1982.

[5] F. M. Henderson and Z. G. Xia, "Radar applications in urban analysis, settlement detection and population estimation," in Manual of Remote Sensing, 3rd ed, vol. 2. New York: Wiley, 1999, pp. 759-761.

[6] A. R. Brenner and L. Roessing, "Radar imaging of urban areas by means of very high-resolution SAR and interferometric SAR," IEEE Trans. Geosci. Remote Sens., vol. 46, no. 10, pp. 2971-2982, Oct. 2008.

[7] C. Tison, J. M. Nicolas, F. Tupin, and H. Maître, "A new statistical model for Markovian classification of urban areas in high-resolution SAR images," IEEE Trans. Geosci. Remote Sens., vol. 42, no. 10, pp. 2046-2057, Oct. 2004.

[8] T. M. Pellizzeri, P. Gamba, P. Lombardo, and F. Dell'Acqua, "Multitemporal/multiband SAR classification of urban areas using spatial analysis: Statistical versus neural kernel-based approach," IEEE Trans. Geosci. Remote Sens., vol. 41, no. 10, pp. 2338-2353, Oct. 2003.

[9] B. N. Haack, "L- and X-band like- and cross-polarized synthetic aperture radar for investigating urban environments," Photogramm. Eng. Remote Sens., vol. 50, pp. 331-340, 1984.

[10] Y. Dong, B. Forster, and C. Ticehurst, "Radar backscatter analysis for urban environments," Int. J. Remote Sens., vol. 18, no. 6, pp. 1351-1364, Apr. 1997.

[11] H. Kimura, K. P. Papathanassiou, and I. Hajnsek, "Polarization orientation effects in urban areas on SAR data," in Proc. IGARSS, 2005, pp. 4863-4867.

[12] Z. G. Xia and F. M. Henderson, "Understanding the relationships between radar response patterns and the bio- and geophysical parameters of urban areas," IEEE Trans. Geosci. Remote Sens., vol. 35, no. 1, pp. 93-101, Jan. 1997.

[13] D. J. Weydahl, "Identifying urban features using RADARSAT images taken at multiple incidence angles," in Proc. IGARSS, 1997, pp. 287-289.

[14] C. Corbane, F. Faure, N. Baghdadi, N. Villeneuve, and M. Petit, "Rapid urban mapping using SAR/optical imagery synergy," Sensors, vol. 8, no. 11, pp. 7125-7143, 2008.

[15] X. Descombes, M. Sigelle, and F. Prêteux, "Estimating Gaussian Markov random field parameters in a nonstationary framework: Application to remote sensing imaging," IEEE Trans. Image Process., vol. 8, no. 4, pp. 490-503, Apr. 1999.

[16] A. Lorette, X. Descombes, and J. Zerubia, "Texture analysis through a Markovian modelling and fuzzy classification: Application to urban area extraction from satellite images," Int. J. Comput. Vis., vol. 36, no. 3, pp. 221-236, Feb./Mar. 2000.

[17] A. Lorette, "Analyse de texture par méthodes Markoviennes et par morphologie mathématique: Application a l'analyse des zones urbaines sur des images satellitales," Ph.D. dissertation, Univ. Nice Sophia Antipolis, Nice, France, 1999.

[18] S.-T. Wu and S. A. Sader, "Multipolarization SAR data for surface feature delineation and forest vegetation characterization," IEEE Trans. Geosci. Remote Sens., vol. GRS-25, no. 1, pp. 67-76, Jan. 1987.

[19] Y. A. Hussin, "Effect of polarization and incidence angle on radar return from urban features using L-band aircraft radar data," in Proc. IGARSS, 1995, pp. 178-180. 